\title{
ACT: A leaf BRDF model taking into account the azimuthal anisotropy of monocotyledonous leaf surface
}

\author{
Alexis Comar a,b,*, Frédéric Baret ${ }^{\mathrm{b}}$, Gaël Obein ${ }^{\mathrm{d}}$, Lionel Simonot ${ }^{\mathrm{e}}$, Daniel Meneveaux ${ }^{\mathrm{e}}$, \\ Françoise Viénot ${ }^{\mathrm{c}}$, Benoit de Solan ${ }^{\mathrm{a}, \mathrm{b}}$ \\ a INRA, UMR EMMAH, UMT CAPTE, Avignon, France \\ ${ }^{\mathrm{b}}$ Arvalis Institut du Végétal, Paris, France \\ c Muséum National d'Histoire Naturelle, CRCC, Paris, France \\ d Laboratoire Commun de Métrologie LNE-CNAM, La Plaine Saint Denis, France \\ e Université de Poitiers, SP2MI, Chasseneuil, France
}

\section{A R T I C L E I N F O}

\section{Article history:}

Received 20 June 2013

Received in revised form 27 November 2013

Accepted 4 December 2013

Available online $\mathrm{xxxx}$

\section{Keywords:}

Sorghum

Wheat

Leaf

Surface roughness

BRDF

BRF

Reflectance

Conoscope

Azimuthal anisotropy

Optical properties

Goniometer

Physical model

Refractive index

\begin{abstract}
A B S T R A C T
Leaf reflectance of monocotyledons generally displays a strong azimuthal anisotropy due to the longitudinal orientation of the veins. The Cook and Torrance (CT) bidirectional reflectance distribution function model was adapted to account for this distinctive feature. The resulting ACT (Anisotropic Cook and Torrance) model is based on the decomposition of the roughness parameter into two perpendicular components. It is evaluated on sorghum (Sorghum halepense) and wheat (Triticum durum) leaf BRF (Bidirectional Reflectance Factor) measurements acquired using a conoscope system. Results show that the ACT model fits the measurements better than azimuthally isotropic surface models: the root mean square error computed over all the BRF measurements for both leaves decreases from $\approx 0.06$ for the Lambertian model to $\approx 0.04$ for the CT model and down to $\approx 0.03$ for the ACT model. The adjusted value of the refraction index is plausible $(n \approx 1.32)$ for both leaves while the retrieved roughness values perpendicular to the veins ( sorghum $=0.56$; wheat $=0.46$ ) is about two times larger than that parallel to the veins (sorghum $=0.27$; wheat $=0.18$ ). Nonetheless, the observed residual discrepancies between the ACT model simulations and the measurements may be explained mainly by the Lambertian assumption of the volume scattering.
\end{abstract}

(c) 2014 Elsevier Inc. All rights reserved.

\section{Introduction}

Leaves depart from the perfect Lambertian behavior as reported by a number of studies (Brakke, Smith, \& Harnden, 1989; Breece Iii \& Holmes, 1971; Howard, 1971). Most leaves, in fact, scatter light in the specular direction, particularly for near grazing illumination directions, because a significant fraction of the incoming light is directly reflected by the epidermis. This fraction of light doesn't interact with the leaf volume (interior) and therefore does not acquire information on leaf biochemical composition (Comar, Baret, Viénot, de Yan, \& Solan, 2012). Knowledge of the leaf bidirectional reflectance distribution function (BRDF) is therefore critical to optimize the measurement configuration for estimating leaf biochemical composition from their

\footnotetext{
* Corresponding author at: INRA Centre de Recherche PACA, UMR 114 EMMAH INRAUAPV, 228 route de l'Aérodrome, CS 40509, Domaine Saint Paul - Site Agroparc, 84914 AVIGNON Cedex 9. Tel.: + 33432722418

E-mail address: alexis.comar@avignon.inra.fr (A. Comar).
}

optical properties, or when using close range imaging techniques at the canopy level to infer leaf biochemical composition (Vigneau, Ecarnot, Rabatel, \& Roumet, 2011). In addition, remote sensing estimates of canopy characteristics should also be dependent on leaf directional properties. Unfortunately, studies in regard to the effect of leaf BRDF on canopy reflectance remain scarce and contradictory: Stuckens, Somers, Delalieux, Verstraten, and Coppin (2009), using canopy radiative transfer model simulations, demonstrated that significant differences could be observed over citrus canopy reflectance when considering a directional component for leaves as defined by Bousquet, Lachérade, Jacquemoud, and Moya (2005). By contrast, Chelle (2006) has shown that the Lambertian assumption for leaves is acceptable when simulating the light absorbed by a wheat canopy as compared to an azimuthally isotropic leaf BRDF assumption. In a review on 3D canopy modeling for remote sensing applications, Lewis (2007) concluded that the accuracy of canopy reflectance simulations suffered from a lack of knowledge on leaf directional properties.

The measurement of leaf directional properties is complex and needs sophisticated devices such as a gonio-reflectometer (e.g. Combes 
et al., 2007; Woolley, 1971), an imaging sphere (Sarto, Woldemar, \& Vanderbilt, 1990), or a conoscope (Comar et al., 2012). Other existing systems could be used (Marschner, Westin, Lafortune, \& Torrance, 2000), but no results have been reported in the literature for leaves. Both illuminating and viewing directions should be varied to describe all BRDF features. Furthermore, Combes et al. (2007) and Comar et al. (2012) demonstrated that the parallel vascular system of monocot leaves induced an oriented roughness pattern that affects the leaf reflectance when the azimuth of the measurement configuration changes. Models account for these features in describing the leaf surface BRDF are thus needed for understanding the canopy reflectance as well as accessing in a more robust way the leaf biochemical composition. The use of physically based models is highly desired as they describe the BRDF over all the directions of illumination and observation using a limited number of meaningful physical parameters. Moreover, these parameters can be adjusted over a sample of bidirectional reflectance measurements.

BRDF models have been intensively studied for computer graphic applications. A number of physically based models consider the surface as a statistical arrangement of micro-facets with the same properties and with a given distribution of their orientation. The microfacets are assumed to be either Lambertian (Oren \& Nayar, 1994) or completely specular, as described by the Fresnel equation (Cook \& Torrance, 1981; Torrance \& Sparrow, 1967). More recently, Simonot (2009) proposed a BRDF model with facets having both Lambertian and specular components. Apart from the behavior of individual facets, their arrangement on the leaf surface creates shadowing and masking effects that contribute to shape the leaf BRDF. Blinn (1977) proposed micro-facets arranged in V groove patterns to simplify the Smith's (1967) shadowing/masking expression derived from Beckmann and Spizzichino (1963). Ashikmin, Premoze and Shirley (2000) proposed an elegant method for linking the micro-facet arrangement with the shadowing/masking term. However, very few BRDF models consider azimuthally anisotropic surfaces such as those observed over monocot leaves. The Ward (1992) model was one of the first to account explicitly for azimuthally anisotropic surfaces. It is based on an approximation of the Cook and Torrance (1981) model (named CT hereafter) where the Fresnel and shadowing/masking terms are merged. Kurt, SzirmayKalos, and Kvrivánek (2010) developed a first "physically plausible" BRDF model based on Ward's work, but it appears inappropriate for leaf BRDF modeling as it considers material having several specular lobes such as car paints.

Bousquet et al. (2005) adjusted the CT physical model parameters to leaf BRDF measurements. They observed a strong agreement with the BRF (Bidirectional Reflectance Factor) measured with a gonioreflectometer over 3 dicotyledonous leaves showing no anisotropic roughness features.

Thus, the literature is very poor regarding BRDF modeling of plant leaves, particularly in the case of azimuthally anisotropic surface roughness.

The objective of this paper is to develop and evaluate a physically based BRDF model able to describe monocot leaf BRF with an azimuthally anisotropic surface. In the first part, the CT model is adapted to the azimuthally anisotropic distribution of the micro-facets based on Ward's (1992) formulation of Beckmann and Spizzichino (1963) micro-facet distribution. The resulting model named ACT (Anisotropic Cook and Torrance) is then adjusted over BRF measurements of wheat and sorghum leaves performed in the visible spectral domain. Finally, the accuracy of the BRF simulations of the ACT model is compared to that of the CT and the Lambertian models with due attention to the adjusted parameters.

\section{Model development}

At a given wavelength, the leaf $B R D F, B R D F_{\text {tot }}$, is generally considered as the sum of a diffuse component corresponding to the scattering within the leaf volume, $B R D F_{d i f f}$, and a directional component, $B R D F_{\text {spec }}$ that originates from the light reflected at the leaf surface:

$B R D F_{\text {tot }}\left(\theta_{i}, \varphi_{i} ; \theta_{r}, \varphi_{r} ; \lambda\right)=B R D F_{\text {spec }}\left(\theta_{i}, \varphi_{i} ; \theta_{r}, \varphi_{r}\right)+B R D F_{\text {diff }}(\lambda)$

where $\theta$ and $\varphi$ are the zenith and azimuth angles, subscripts $i$ and $r$ refer to the incoming and reflected directions (Fig. 1), and $\lambda$ refers to the wavelength. The term $B R D F_{\text {diff }}$ is assumed to be Lambertian and to only depend on the wavelength. Conversely, the $B R D F_{\text {spec }}$ term depends on the geometrical configuration and is assumed to be wavelength independent: in actuality, specular reflection varies as a function of the facet orientation and the refractive index, which is marginally variable in the visible domain.

Cook and Torrance (1981), followed by Bousquet et al. (2005), assume that the $B R D F_{\text {spec }}$ term originates from the contribution of micro-facets that have a mirror-like behavior in that they reflect light in the specular direction. The half angle between the incoming and reflected directions, $\theta_{h}$ is given by

$\cos \left(2 \theta_{h}\right)=\cos \left(\theta_{i}\right) \cos \left(\theta_{r}\right)+\sin \left(\theta_{i}\right) \sin \left(\theta_{r}\right)\left[\cos \left(\varphi_{i}\right) \cos \left(\varphi_{r}\right)+\sin \left(\varphi_{i}\right) \sin \left(\varphi_{r}\right)\right]$.

All the same surface area noted $a$ with an orientation described by a probability function $\mathrm{P}(\alpha, \beta)$ (also expressed as $\mathrm{P}\left(\omega_{h}\right)$ ) where $\alpha$ and $\beta$ are, respectively, the zenith and the azimuth angles of the normal of the micro-facet, and $\omega_{h}$ is the solid angle including the micro-facets concerned by the reflexion (Fig. 1). A probability function respects the following properties:

$\int_{2 \pi} \mathrm{P}\left(\omega_{h}\right) \mathrm{d} \omega_{h}=\int_{0}^{2 \pi} \int_{0}^{\pi / 2} \mathrm{P}(\alpha, \beta) \sin (\alpha) \mathrm{d} \alpha \mathrm{d} \beta=1$.

The micro-facets contributing to the reflection in the direction $\left[\theta_{r}, \varphi_{r}\right]$ have their normal pointing along the bisector between the incident and the reflection directions. Their orientation is linked to the illumination and view directions (Fig. 1):

$$
\begin{array}{ll}
\alpha\left(\theta_{i}, \varphi_{i} ; \theta_{r}, \varphi_{r}\right)= & \cos ^{-1}\left(\frac{\cos \left(\theta_{i}\right) \cdot \cos \left(\theta_{r}\right)}{2 \cdot \cos \left(\theta_{h}\right)}\right) \\
\beta\left(\theta_{i}, \varphi_{i} ; \theta_{r}, \varphi_{r}\right)= & \tan ^{-1}\left(\frac{\sin \left(\theta_{i}\right) \sin \left(\varphi_{i}\right)+\sin \left(\theta_{r}\right) \sin \left(\varphi_{r}\right)}{\sin \left(\theta_{i}\right) \cos \left(\varphi_{i}\right)+\sin \left(\theta_{r}\right) \cos \left(\varphi_{r}\right)}\right) .
\end{array}
$$

The number, $n_{\mu}\left(\theta_{i}, \varphi_{i} ; \theta_{r}, \varphi_{r}\right)$, of micro-facets contributing to the reflection for incidence $\left[\theta_{i}, \varphi_{i}\right]$ and reflected $\left[\theta_{r}, \varphi_{r}\right]$ directions is:

$$
n_{\mu}\left(\theta_{i}, \varphi_{i} ; \theta_{r}, \varphi_{r}\right)=P\left(\omega_{h}\right) \delta \omega_{h} n_{t o t}
$$

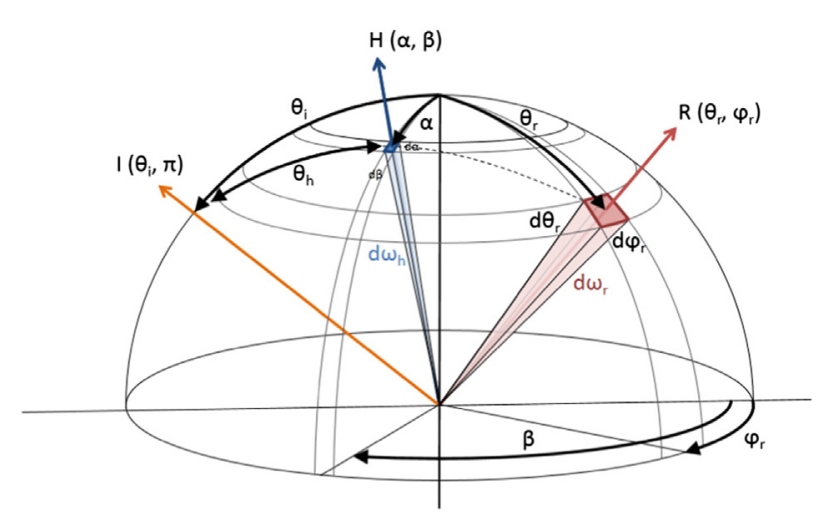

Fig. 1. Geometrical configuration of the reflectance measurements. Directions I and R correspond to the incidence and reflected directions. Direction $\mathrm{H}$ corresponds to the normal of the micro-facet. 
where $n_{\text {tot }}$ is the total number of micro-facets within the surface $S$. The flux, $\Phi_{\mu}\left(\theta_{h}\right)$, received by each micro-facet is:

$\Phi_{\mu}\left(\theta_{h}\right)=a E_{0} \cos \left(\theta_{h}\right)$

where $E_{0}$ is the irradiance for normal incidence $\left(\theta_{i}=0\right)$. The radiance of the illuminated and sampled leaf surface, $S$, is therefore:

$L_{r}\left(\theta_{i}, \varphi_{i} ; \theta_{r}, \varphi_{r}\right)=\frac{\mathrm{P}(\alpha, \beta) \delta \omega_{h} n_{t o t} a E_{0} \cos \left(\theta_{h}\right)}{S \cos \left(\theta_{r}\right) \delta \omega_{r}} \operatorname{SM}\left(\theta_{i}, \varphi_{i} ; \theta_{r}, \varphi_{r}\right) F\left(n, \theta_{h}\right)$

where $\omega_{r}$ is the solid angle of the receiver beam (Fig. 1 ), $S M\left(\theta_{i}, \varphi_{i} ; \theta_{r}, \varphi_{r}\right)$ is the shadowing/masking term that accounts for the interactions between micro-facets; $F\left(n, \theta_{h}\right)$ is the Fresnel term for dielectric materials characterized by the refraction index, $n$, and for non-polarized incident light (Fresnel, 1868; Sparrow \& Cess, 1978):

$F\left(n, \theta_{h}\right)=\frac{1}{2} \cdot\left(\frac{G-C}{G+C}\right)^{2} \cdot\left[1+\frac{(C \cdot(G+C)-1)^{2}}{(C \cdot(G+C)+1)^{2}}\right]$

with: $G=n^{2}+C^{2}-1$ and $C=\cos \left(\theta_{h}\right)$.

Rense (1950), followed by Nayar, Ikeuchi, and Kanade (1991) and Papetti, Walker, Keffer and Johnson (2007), demonstrated that

$\delta \omega_{r}=4 \cos \left(\theta_{h}\right) \delta \omega_{h}$.

The surface $S$ is linked to the number of micro-facets $n_{\text {tot }}$, their area and their orientation:

$S=\int_{0}^{2 \pi} \int_{0}^{\pi / 2} a n_{\text {tot }} P(\alpha, \beta) \cos (\alpha) \sin (\alpha) d \alpha d \beta=a n_{\text {tot }} P_{\text {int }}$

with:

$P_{\text {int }}=\int_{0}^{2 \pi} \int_{0}^{\pi / 2} P(\alpha, \beta) \cos (\alpha) \sin (\alpha) d \alpha d \beta$.

Finally, using Eqs. (9) and (10), the expression of the radiance in Eq. (7) gives a simplified expression of the radiance of the illuminated sampled area:

$L_{r}\left(\theta_{i}, \varphi_{i} ; \theta_{r}, \varphi_{r}\right)=\frac{\mathrm{P}(\alpha, \beta)}{P_{i n t}} \frac{E_{0}}{4 \cos \left(\theta_{r}\right)} \operatorname{SM}\left(\theta_{i}, \varphi_{i} ; \theta_{r}, \varphi_{r}\right) F\left(n, \theta_{h}\right)$.

The BRDF of the surface due to specular reflection by the microfacets is given by:

$B R D F_{\text {spec }}\left(\theta_{i}, \varphi_{i} ; \theta_{r}, \varphi_{r}\right)=\frac{L_{r}\left(\theta_{i}, \varphi_{i} ; \theta_{r}, \varphi_{r}\right)}{E_{i}\left(\theta_{i}, \varphi_{i}\right)}=\frac{L_{r}\left(\theta_{i}, \varphi_{i} ; \theta_{r}, \varphi_{r}\right)}{E_{0} \cos \left(\theta_{i}\right)}$

where $E_{i}\left(\theta_{i}, \varphi_{i}\right)$ is the irradiance received by the illuminated and sampled area. This provides the general formulation of the micro-facet specular model:

$B R D F_{\text {spec }}\left(\theta_{i}, \varphi_{i} ; \theta_{r}, \varphi_{r}\right)=\frac{P(\alpha, \beta)}{P_{\text {int }}} \frac{1}{4 \cos \left(\theta_{r}\right) \cos \left(\theta_{i}\right)} \operatorname{SM}\left(\theta_{i}, \varphi_{i} ; \theta_{r}, \varphi_{r}\right) F\left(n, \theta_{h}\right)$.

Several micro-facet distributions can be found in the literature that describe $P(\alpha, \beta)$. The distribution proposed by Beckmann and Spizzichino (1963) and used by Bousquet et al. (2005) was selected and can be expressed as:

$P(\alpha, \beta)=\frac{P_{\text {int }}}{\pi \sigma^{2} \cos ^{4}(\alpha)} \exp \left(-\left(\frac{\tan (\alpha)}{\sigma}\right)^{2}\right)$

where $\sigma$ is the roughness parameter. The probability of micro-facet appearance $P(\alpha, \beta)$ defined in this study and the distribution function
$D(\alpha, \beta)$ found in Cook and Torrance (1981) or Bousquet et al. (2005) are linked through the normalization factor $P_{\text {int }}$ :

$D(\alpha, \beta)=\frac{P(\alpha, \beta)}{P_{\text {int }}}$.

Eq. (15) describes azimuthally isotropic surfaces. The principles proposed by Ward (1992) were used to extend the Beckmann distribution to azimuthally anisotropic distributions by considering two perpendicular and uncorrelated roughness parameters $\left(\sigma_{x}, \sigma_{y}\right)$ :

$P(\alpha, \beta)=\frac{P_{\text {int }}}{\pi \sigma_{x} \sigma_{y} \cos ^{4}(\alpha)} \exp \left(-\tan ^{2}(\alpha)\left(\frac{\cos ^{2}(\beta)}{\sigma_{x}^{2}}+\frac{\sin ^{2}(\beta)}{\sigma_{y}^{2}}\right)\right)$.

Note that this expression verifies Eq. (3) and the radial symmetry expected for such a surface:

$P(\alpha, \beta)=P(\alpha, \beta+\pi)$ with $\alpha \epsilon\left[0, \frac{\pi}{2}\right]$ and $\beta \epsilon[0,2 \pi]$.

Moreover if $\left(\sigma_{x}=\sigma_{y}\right)$ then $P(\alpha, \beta)$ defined by Eq. (17) simplifies into Eq. (15), which shows that the ACT model reduces to the CT model for anisotropic roughness.

The micro-facets are arranged in $\mathrm{V}$ shaped grooves with the sides equal and oriented symmetrically with regard to the leaf surface normal as suggested by Torrance and Sparrow (1967). This allows using the Blinn (1977) shadowing/masking factor.

$S M=\min \left(1, E_{1}, E_{2}\right)$ with $E_{1}=\frac{2 \cos (\alpha) \cos \left(\theta_{r}\right)}{\cos \left(\theta_{h}\right)}$ and $E_{2}=\frac{2 \cos (\alpha) \cos \left(\theta_{i}\right)}{\cos \left(\theta_{h}\right)}$

\section{Materials and methods}

\subsection{Reflectance measurements}

The BRF measurements of wheat leaves (Triticum durum) come from a previous study (Comar et al., 2012). Sorghum leaves (Sorghum halepense), cultivated in the garden of the Muséum national d'histoire naturelle of Paris, were sampled using the same protocol. In both cases an EZ Contrast 80M (www.eldim.fr) was used to measure the BRF of wheat and sorghum leaves. Measurements were performed for viewing zenith angles in the range of $0^{\circ}$ to $80^{\circ}$ and for all azimuth angles with $1^{\circ}$ step. The illumination zenith angle was fixed at $\theta_{i}=45^{\circ}$ while the incident azimuth was either parallel $\left(\varphi_{i}=0^{\circ}\right)$ or perpendicular $\left(\varphi_{i}=90^{\circ}\right)$ to the veins of the leaf as illustrated in Fig. 2A. For wheat, 5 leaves with 3 measurements on each leaf were collected, leading to 15 separate acquisitions. For sorghum, only one leaf was sampled with 10 measurements collected at different locations on the leaf. The dataset recorded in this study for each species was acquired in about 2 h. No leaf damage was observed during the experiment. The expected symmetry along the incident direction was used to artificially double the sampling, making respectively 30 and 20 replicates for wheat and sorghum leaf measurements.

The light reflected by the leaf sample was measured in three broad spectral bands approaching the color matching functions $\bar{x}(\lambda), \bar{y}(\lambda)$ and $\bar{z}(\lambda)$ of the CIE 1931 standard colorimetric observer: $\bar{x}(\lambda), \bar{y}(\lambda)$ and $\bar{z}(\lambda)$ are respectively located in the blue, green and red parts of the visible domain (Fig. 2B). More details can be found in Comar et al. (2012).

The relative radiance measurements were thus transformed into Bidirectional Reflectance Factor (BRF) (Nicodemus, Richmond, Hsia, Ginsberg \& Limperis, 1977) values using a calibration coefficient $\gamma$ :

$B R F_{\text {leaf }}\left(45^{\circ}, \varphi_{i}, \theta_{r}, \varphi_{r}, \lambda\right)=\gamma S_{\text {leaf }}\left(45^{\circ}, \varphi_{i}, \theta_{r}, \varphi_{r}, \lambda\right)$

where $S_{\text {leaf }}$ is the signal measured by the system. Because of the small solid angles under which the leaves were illuminated and viewed, the 
B
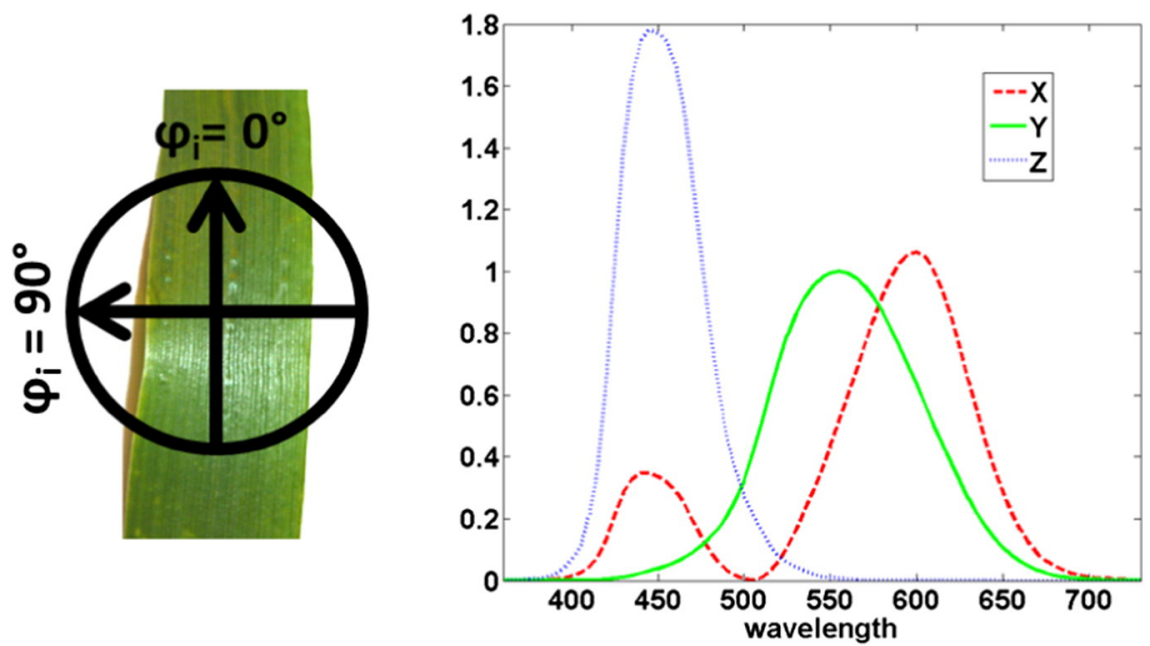

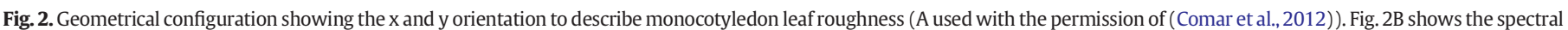

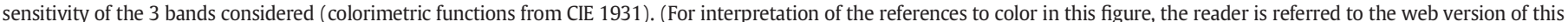
article.)

BRF measurements will be approximated as related to the modeled $B R D F$ according to:

$B R F=\pi B R D F$

The calibration coefficient $\gamma$ was computed by comparing it with a reference spectralon surface with known directional hemispherical reflectance factor (DHRF). The DHRF at $\theta_{i}=8^{\circ}$ view zenith angle, of the spectralon reference surface was provided by Labsphere (www. labsphere.com) and the US National Institute of Standards and Technology (NIST) in the visible domain $(400 \mathrm{~nm}<\lambda<700 \mathrm{~nm}): D H R F_{\text {ref }}\left(\theta_{i}=\right.$ $\left.8^{\circ}, \lambda\right)=0.991$. Following Weidner and Hsia (1981), it is assumed that the DHRF of the spectralon surface is stable when the incident light decreases to $\theta_{i}=45^{\circ}$ (no change in absorption):

$D H R F_{\text {ref }}\left(\theta_{i},=45^{\circ}, \varphi_{i}, \lambda\right) \approx D H R F_{r e f}\left(\theta_{i}=8^{\circ}, \lambda\right)=0.991$

The $\operatorname{DHRF}_{r e f}\left(45^{\circ}, \varphi_{i}, \lambda\right)$ corresponds to the integration of the BRDF over the hemisphere:

$D H R F_{r e f}\left(\theta_{i}, \varphi_{i}, \lambda\right)=\int_{0}^{2 \pi} \int_{0}^{\pi / 2} B R D F\left(\theta_{i}, \varphi_{i}, \theta_{r}, \varphi_{r}, \lambda\right) \sin \left(\theta_{r}\right) \cos \left(\theta_{r}\right) \mathrm{d} \theta_{r} \mathrm{~d} \varphi_{r}$.

It may be computed numerically using the measured reflected signal, $S_{r e f}\left(45^{\circ}, \varphi_{i}, \theta_{r}, \varphi_{r}, \lambda\right)$ :

$\operatorname{DHRF}_{\text {ref }}\left(45^{\circ}, \varphi_{i}, \lambda\right)=\gamma \frac{\sum_{\varphi r=0^{\circ}}^{360^{\circ}} \sum_{\theta r=0^{\circ}}^{90^{\circ}} S_{r e f}\left(45^{\circ}, \varphi_{i}, \theta_{r}, \varphi_{r}, \lambda\right) \sin \left(\theta_{r}\right) \cos \left(\theta_{r}\right)}{\sum_{\varphi r=0^{\circ}}^{360^{\circ}} \sum_{\theta r=0^{\circ}}^{90^{\circ}} \sin \left(\theta_{r}\right) \cos \left(\theta_{r}\right) .}$

Because of the absence of measurements for $\theta_{i}>80^{\circ}$, the integration over the entire range of view zenith angles in Eq. (24) was computed assuming a linear variation with $\theta r$ of the term $S_{r e f}\left(45^{\circ}, \varphi_{i}, \theta_{r}, \varphi_{r}, \lambda\right) \sin \left(\theta_{r}\right)$ $\cos \left(\theta_{r}\right)$ for $80^{\circ}<\theta_{i}<90^{\circ}$, taking advantage of the property: $\cos \left(90^{\circ}\right)=0$. Note that Eq. (24) was also applied to the leaf measurements to compute $\operatorname{DHRF}_{\text {leaf }}\left(45^{\circ}, \varphi_{i}, \lambda\right)$, which was used as one metric to characterize leaf BRF patterns.

\subsection{Model adjustment to observations}

The BRDF model described in Eq. (1) with a specular component corresponding to Eq. (14) was adjusted to the BRF measurements available, tuning the set of parameters $\left[n, \sigma_{x}, \sigma_{y}, D H R F_{\text {diff }}\right]$ for minimizing a cost function.

The cost function based on the root mean square error (RMSE) was minimized separately for each waveband using the simplex algorithm (Nelder \& Mead, 1965) and implemented in the Matlab software. This allows for verifying that the surface parameters $\left[n, \sigma_{x}, \sigma_{y}\right]$ are independent from the wavelength.

The cost function, $R M S E_{x}$, reflects the quadratic difference between measurements and model simulations computed over the 57,600 available directions (28,800 directions times two illumination azimuth angles)

$$
\operatorname{RMSE}_{x}=\sqrt{\frac{\left.\sum_{\varphi_{\mathrm{i}}=[0} 9_{0}^{\circ}\right] \sum_{\varphi_{\mathrm{r}}=0.5}^{359.5} \sum_{\theta_{\mathrm{r}}=0.5}^{79.5} w_{x}(B R F-B R F)^{2}}{\sum_{\varphi_{i}=\left[090^{\circ}\right]} \sum_{\varphi_{\mathrm{r}}=0.5}^{359.5} \sum_{\theta_{\mathrm{r}}=0.5}^{79.5} w_{x}}}
$$

where $w_{x}$ is a weighting factor. To improve the sensitivity to the directional features that are observed mainly for the medium to high zenith view angles, we considered the weighting $w_{f i t}=\sin \left(\theta_{r}\right)$ corresponding to $R M S E_{f i t}$ cost function. Two alternative metrics were also computed in order to compare the model simulations with the observations, but were consequently not used in the minimization process: $R M S E_{i s o}$, which considers an equal weighting of all observations ( $w_{\text {iso }}=1$ ), pertinent in the case of a sampling as proposed by Fliege and Maier (1999); and $R M S E_{\text {hem }}$, where $w_{\text {hem }}=\cos \left(\theta_{r}\right) \sin \left(\theta_{r}\right)$, which considers the weighting used in the DHRF angular integration (Eq. (24)), with a maximum weight for $\theta_{r}=45^{\circ}$.

\section{Results and discussion}

The BRF directional patterns of sorghum and wheat leaves are first described with emphasis on the contribution of several components. Then, the ACT model performances are presented and compared to the simple Lambertian (SL) model and CT.

\subsection{Sorghum and wheat leaf BRDF features}

The BRDF features of sorghum (Fig. 3) are similar to those described by Comar et al. (2012) on wheat leaves. A strong specular lobe is observed in the specular direction when the leaf is illuminated in a direction perpendicular to the veins $\left(\varphi_{i}=90^{\circ}\right)$. Conversely, the specular lobe is less pronounced but much broader when illuminating the leaf in a direction parallel to the veins $\left(\varphi_{i}=0^{\circ}\right)$. This can be explained by 

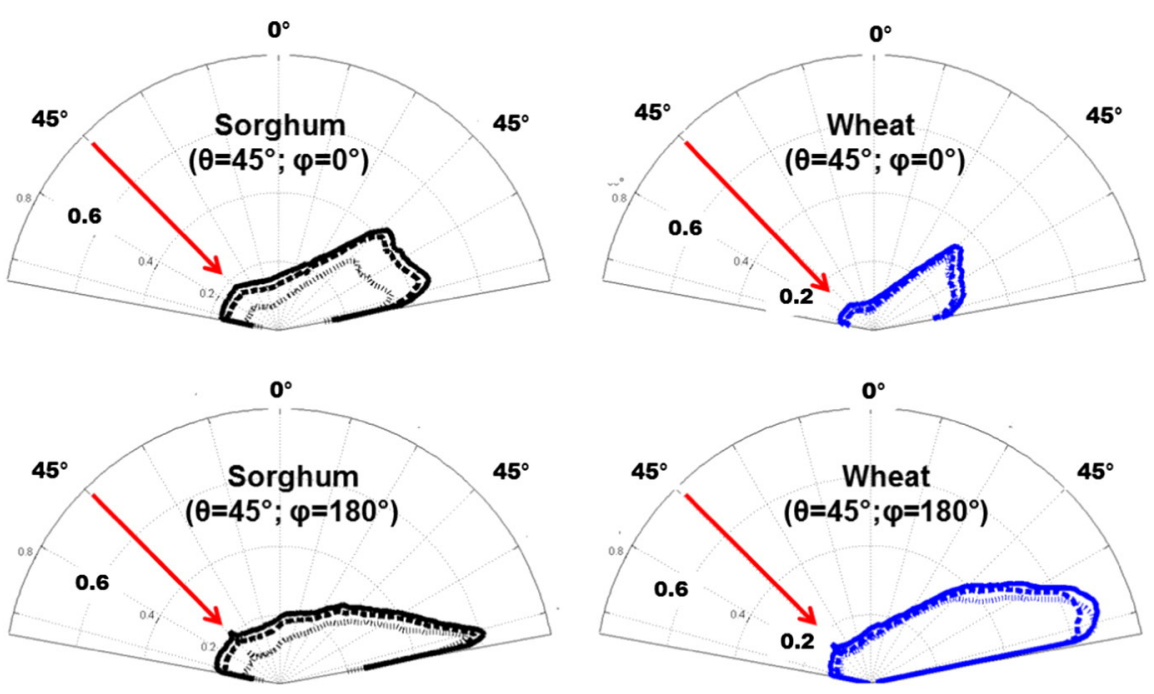

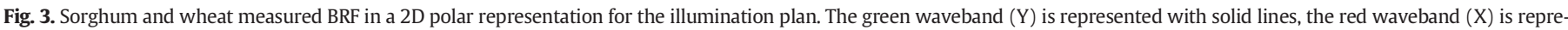

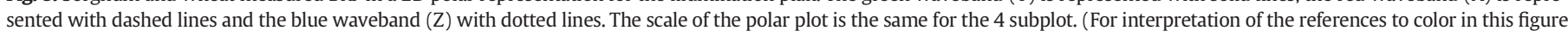
legend, the reader is referred to the web version of this article.)

the distribution of the orientation of the micro-facets: when parallel to the veins, the incoming light is reflected by each side of the veins, producing a specular lobe that spans over a broad directional domain in azimuth (Fig. 4). Conversely, when the veins are perpendicular to the illumination direction, most of the light is reflected by the 'top' of the veins, creating a very narrow specular lobe. These results are consistent with the previous observations on monocot leaves (Comar et al., 2012; Combes et al., 2007). Note that the maximum BRF value is observed in the specular direction $\left(\theta_{r}=45^{\circ}\right)$ for parallel illumination $\left(\varphi_{i}=0^{\circ}\right)$, while the maximum reflectance is achieved at $70^{\circ}<\theta_{r}<77^{\circ}$ for perpendicular illumination $\left(\varphi_{i}=90^{\circ}\right)$. This is due to the strong enhancement of the specular reflection for grazing angles on the micro-facets observed in this illumination situation.

The hot-spot is more pronounced for illumination perpendicular to the veins $\left(\varphi_{i}=90^{\circ}\right)$ because of the shadowing effects by the veins. Conversely, for illumination parallel to the veins $\left(\varphi_{i}=0^{\circ}\right)$, almost no hot-spot feature is observed because no shadowing effect by the veins is expected (Fig. 3).

The reflectance of wheat leaves is generally lower than that of sorghum leaves, except in the specular direction when illuminated perpendicularly to the veins (Fig. 3). The lower wheat reflectance values associated with a darker appearance might be explained by a larger chlorophyll and carotenoid content in wheat compared to sorghum leaves. This is also consistent with the diffuse component that mainly corresponds to the volume scattering driven by absorption by chlorophyllian pigments. According to Comar et al. (2012), the diffuse component was approximated as the minimum of the BRF values measured, $B R F_{\min }$. To avoid possible local measurement errors, the value of the observed BRF corresponding to the $5 \%$ percentile computed over all directions was used instead of the actual minimum BRF value (Comar et al., 2012). Note that because the diffuse component is assumed to be Lambertian, $B R F_{\min } D H R F_{\text {diff. }}$ The diffuse component,

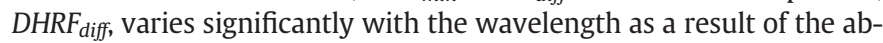
sorption features of leaf pigments including chlorophyll and carotenoids. The $D H R F_{\text {diff }}$ values for a given waveband and a given species show only small variation between the two illumination conditions (Table 1).

The application of Eq. (1) to directional hemispherical reflectance allows to compute the specular component, $D H R F_{\text {spec }}$, as the difference between the $D H R F_{\text {tot }}$ and the $D H R F_{\text {diff. }}$. As expected, results (Table 1) indicate that $D H R F_{\text {spec }}$ is almost independent of waveband in the visible domain. The $D H R F_{\text {spec }}$ values also demonstrate that only slight variation exists between the two illumination conditions for a given waveband and species (Table 1), despite large differences in the directional distribution patterns (Fig. 3). Wheat and sorghum leaves show similar patterns for all illumination directions (Fig. 3). However, the more anisotropic reflectance pattern observed for wheat can be explained mainly by the differences between surface features as seen in the scanning electronic microscope (SEM) images of Fig. 4: wheat leaves are rougher with veins separated by relatively short distances as compared to the sorghum surface that appears smoother due to veins separated by larger distances. In addition, the trichomes observed on wheat leaves may also partly explain the observed differences.

\subsection{ACT model performances}

The model described by Eqs. (1) and (14) as well as models considering azimuthally isotropic roughness, i.e. the simple Lambertian (SL)

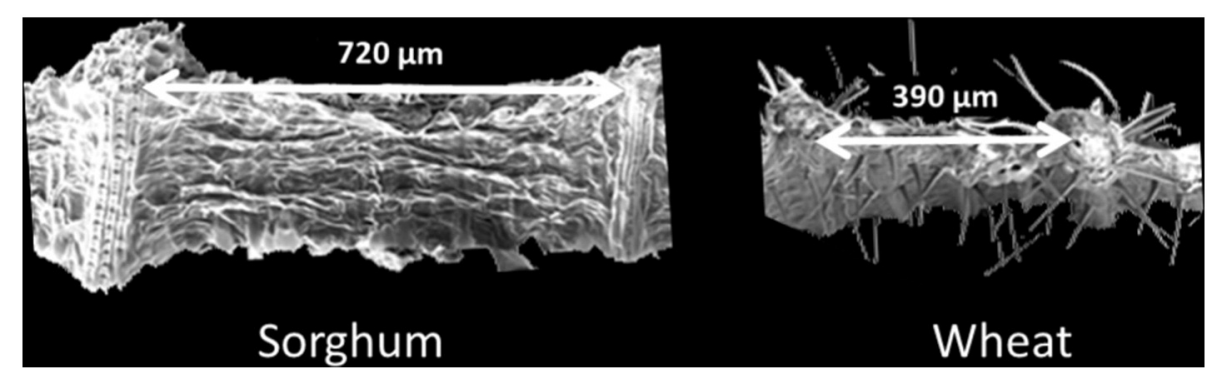

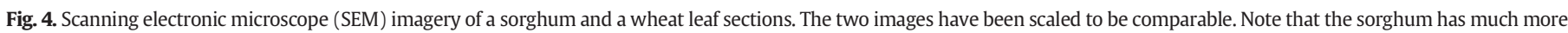
spaced veins and no trichomes as compared to wheat. 
Table 1

Computed DHRF values for the 3 wavebands.

\begin{tabular}{|c|c|c|c|c|c|c|c|}
\hline \multirow[t]{2}{*}{ Feature } & \multirow[t]{2}{*}{ Illumination azimuth } & \multicolumn{3}{|l|}{ Sorghum } & \multicolumn{3}{|l|}{ Wheat } \\
\hline & & $\bar{x}(\lambda)$ red & $\bar{y}(\lambda)$ green & $\bar{z}(\lambda)$ blue & $\bar{x}(\lambda)$ red & $\bar{y}(\lambda)$ green & $\bar{z}(\lambda)$ blue \\
\hline \multirow[t]{2}{*}{$D H R F_{\text {diff }}$} & $\varphi_{i}=0^{\circ}$ & 0.125 & 0.149 & 0.072 & 0.063 & 0.082 & 0.055 \\
\hline & $\varphi_{i}=90^{\circ}$ & 0.141 & 0.164 & 0.086 & 0.076 & 0.095 & 0.067 \\
\hline \multirow[t]{2}{*}{$D_{H R F_{\text {spec }}}$} & $\varphi_{i}=0^{\circ}$ & 0.055 & 0.055 & 0.048 & 0.047 & 0.049 & 0.051 \\
\hline & $\varphi_{i}=90^{\circ}$ & 0.035 & 0.035 & 0.032 & 0.037 & 0.039 & 0.039 \\
\hline \multirow[t]{2}{*}{$D H R F_{t o t}$} & $\varphi_{i}=0^{\circ}$ & 0.180 & 0.204 & 0.120 & 0.110 & 0.131 & 0.106 \\
\hline & $\varphi_{i}=90^{\circ}$ & 0.176 & 0.199 & 0.118 & 0.114 & 0.136 & 0.106 \\
\hline
\end{tabular}

model and the Cook and Torrance (CT) model, were adjusted to the BRF measurements for each species and each waveband independently. Note that none of the three models describe the backscattering hotspot feature nor do previous models found in the literature. As shown previously (Fig. 3), the hotspot is a small and local component that was demonstrated to contribute only marginally to the total DHRF (Comar et al., 2012) and was therefore neglected.

The minimization process for all three models was successfully completed with $R M S E_{f i t}$ values ranging from 0.076 down to 0.033 (Table 2). The ACT model leads to the lowest RMSE $E_{\text {fit }}$ values of 0.041 for sorghum and 0.036 for wheat in the green waveband (Table 2), which corresponds to about $20-30 \%$ of the total leaf DHRF value (Table 1 ). The simple Lambertian model provides $R M S E_{\text {fit }}$ values about twice those of the ACT model, while the CT model has RMSE values $20 \%$ higher than those of the ACT model. This demonstrates the interest in the ACT model compared to the CT and SL models.

Note that the three RMSE values are strongly correlated $\left(R^{2}=0.99\right)$, with $R M S E_{i s o} R M S E_{h e m} 0.88 R^{R M S E} E_{f i t}$ showing that the ranking between models is relatively independent from the applied weighting. However, the fact that the $R M S E_{\text {fit }}$ values are slightly higher than the other RMSE values indicates that the $w_{f i t}=\sin \left(\theta_{r}\right)$ weighting put the emphasis on directions with pronounced features, and justifies a posteriori the selection of the RMSE fit cost function.

Inspection of the ACT model parameters shows as expected, that the estimated refractive index is highly stable across bands and species (Table 2) with an average value close to $n=1.32$, although the fitting of the parameters was conducted independently for each band and each species. Note that the retrieval of this parameter using the CT model yields refractive index of $n=1.4$ for sorghum but $n=1.27$ for wheat, showing possible confounding effects between surface roughness features and the refractive index. It is encouraging that similar refractive indexes were found for both leaves with the inversion of the
ACT model, as this parameter is not expected to vary much between both leaves. The value of $n$ estimated in this study is close to that used within the PROSPECT (Jacquemoud and Baret, 1990) leaf optical properties model $(n \approx 1.4$ ), although it mostly refers to the cell wall material in the mesophyll. This value is also close to $n=1.47$ found by Vanderbilt and Grant (1986) over several leaves using the Brewster angle.

The two roughness component parameters are highly stable across bands (Table 1 ) as a result of very similar specular features in the three bands (Figs. 3 and 5). The roughness perpendicular to the veins $\left(\sigma_{y}\right)$ is about twice that estimated in the direction parallel to the veins $\left(\sigma_{x}\right)$ (Table 2). However, the roughness of the sorghum appears to be larger than that of the wheat even though the SEM images suggest the opposite (Fig. 3). This could tentatively be explained by the presence of trichomes on wheat leaves, which are known to scatter light and to increase leaf reflectance (Levizou et al. 2005). Still, dedicated experiments would be necessary to verify this explanation. Finally, the volume scattering represented by $D H R F_{\text {diff }}$ conspicuously varies with the wavelength, with a minimum in the blue and a maximum in the green (Table 1) in agreement with the known absorption features of chlorophyll and carotenoid pigments. The $D H R F_{\text {diff }}$ of sorghum is larger than that of the wheat (Table 2), consistent with previous observations of the measured $D H R F_{\text {diff }}$ (Table 1 ).

As a result of the general good match of the directional features by the ACT model, the simulated DHRF values also agree strongly with the estimated DHRF values using Comar et al. (2012) method (Fig. 6). The simulated $D H R F_{\text {spec }}$ shows very little variation across wavebands, species, or illumination directions, with a slight underestimation (0.008) when compared to the measured $D H R F_{\text {spec }}$. The $D H R F_{\text {diff }}$ shows a realistic variability across species and bands with a slight overestimation (0.0157), particularly in the case of illumination parallel to the veins $\left(\varphi_{i}=0^{\circ}\right)$. Note that the volume component in the ACT model is

Table 2

Goodness of fit and estimated parameters of the three BRDF models considered in this study.

\begin{tabular}{|c|c|c|c|c|c|c|c|c|}
\hline \multirow[t]{2}{*}{ Model } & \multirow[t]{2}{*}{ Variables properties } & \multirow[t]{2}{*}{ Species } & \multicolumn{3}{|l|}{ Sorghum } & \multicolumn{3}{|l|}{ Wheat } \\
\hline & & & $\bar{x}(\lambda)$ red & $y(\lambda)$ green & $\bar{z}(\lambda)$ blue & $\bar{x}(\lambda)$ red & $\bar{y}(\lambda)$ green & $\bar{z}(\lambda)$ blue \\
\hline \multirow[t]{4}{*}{ SL } & \multirow[t]{3}{*}{ Goodness of fit } & $R M S E_{f i t}$ & 0.071 & 0.073 & 0.066 & 0.072 & 0.076 & 0.074 \\
\hline & & $R M S E_{\text {hem }}$ & 0.064 & 0.065 & 0.058 & 0.066 & 0.069 & 0.067 \\
\hline & & $R_{M S E_{i s o}}$ & 0.064 & 0.065 & 0.058 & 0.063 & 0.066 & 0.065 \\
\hline & Retrieved parameters & $D H R F_{\text {diff }}$ & 0.190 & 0.213 & 0.129 & 0.118 & 0.138 & 0.113 \\
\hline \multirow[t]{6}{*}{$\mathrm{CT}$} & \multirow[t]{3}{*}{ Goodness of fit } & $R M S E_{f i t}$ & 0.045 & 0.046 & 0.038 & 0.044 & 0.047 & 0.046 \\
\hline & & $R_{M S E_{\text {hem }}}$ & 0.039 & 0.040 & 0.033 & 0.038 & 0.040 & 0.039 \\
\hline & & $R_{M S E_{i s o}}$ & 0.041 & 0.042 & 0.035 & 0.039 & 0.041 & 0.040 \\
\hline & \multirow[t]{3}{*}{ Retrieved parameters } & $n$ & 1.42 & 1.44 & 1.40 & 1.26 & 1.27 & 1.27 \\
\hline & & $\sigma$ & 0.506 & 0.516 & 0.498 & 0.225 & 0.229 & 0.250 \\
\hline & & $D H R F_{\text {diff }}$ & 0.151 & 0.173 & 0.093 & 0.095 & 0.114 & 0.088 \\
\hline \multirow[t]{7}{*}{ ACT } & \multirow[t]{3}{*}{ Goodness of fit } & $R M S E_{f i t}$ & 0.040 & 0.041 & 0.033 & 0.034 & 0.036 & 0.033 \\
\hline & & RMSE $_{\text {hem }}$ & 0.033 & 0.034 & 0.028 & 0.028 & 0.029 & 0.027 \\
\hline & & $R_{M S E_{i s o}}$ & 0.037 & 0.038 & 0.030 & 0.029 & 0.031 & 0.029 \\
\hline & \multirow[t]{4}{*}{ Retrieved parameters } & $\mathrm{n}$ & 1.34 & 1.35 & 1.33 & 1.31 & 1.32 & 1.33 \\
\hline & & $\sigma_{\mathrm{x}}$ & 0.264 & 0.269 & 0.270 & 0.182 & 0.184 & 0.194 \\
\hline & & $\sigma_{\mathrm{y}}$ & 0.556 & 0.566 & 0.560 & 0.456 & 0.464 & 0.502 \\
\hline & & $D H R F_{\text {diff }}$ & 0.158 & 0.180 & 0.099 & 0.089 & 0.108 & 0.081 \\
\hline
\end{tabular}




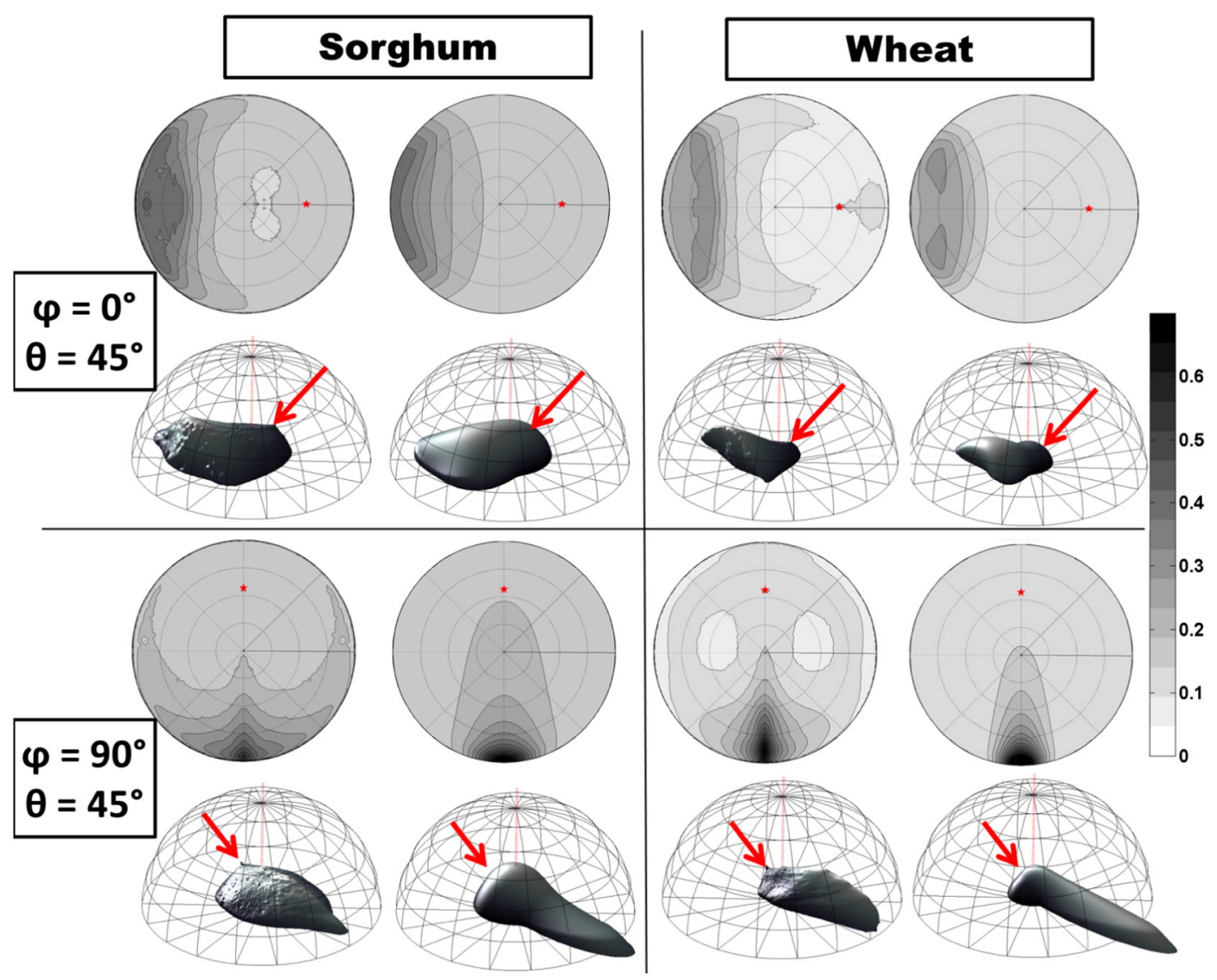

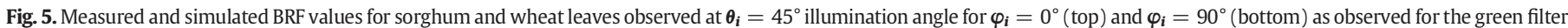

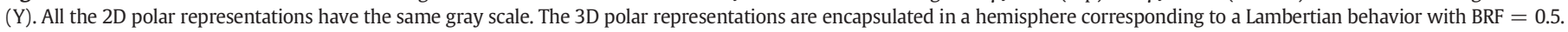

assumed independent from the illumination direction, which may be worth questioning. However, the assumption that $B R F_{\min } D H R F_{\text {diff }}$ may also be questioned.

As a result of the previous findings, the simulated $D H R F_{\text {tot }}$ strongly agrees with the measured ones, with the biases observed for the specular and diffuse components compensating themselves with a residual overestimation of 0.0073 .
Although the model allows for simulating the DHRF components with a relatively high degree of accuracy while describing the main BRDF directional features, Fig. 7 shows that a closer inspection of the discrepancies is needed. When the incidence angle is parallel to the veins $\left(\varphi_{i}=0^{\circ}\right)$, the specular component is underestimated and the diffuse component overestimated (Figs. 5 and 7). By contrast, the specular features offer a better match for perpendicular illumination $\left(\varphi_{i}=90^{\circ}\right)$.
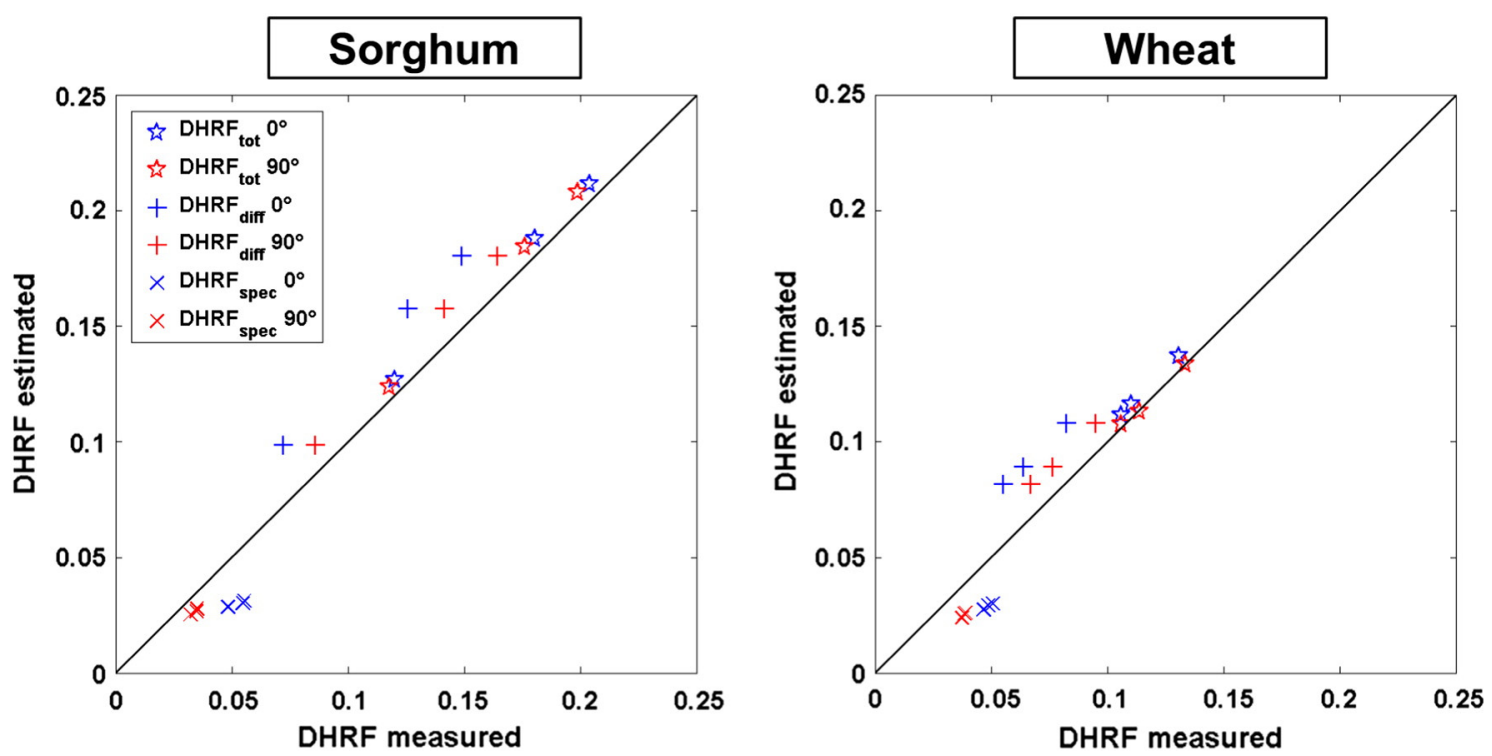

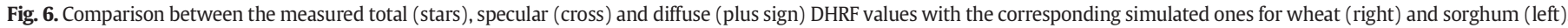

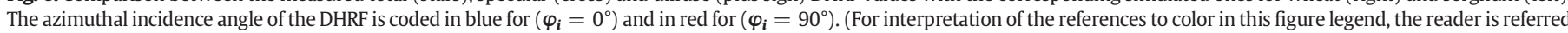
to the web version of this article.) 

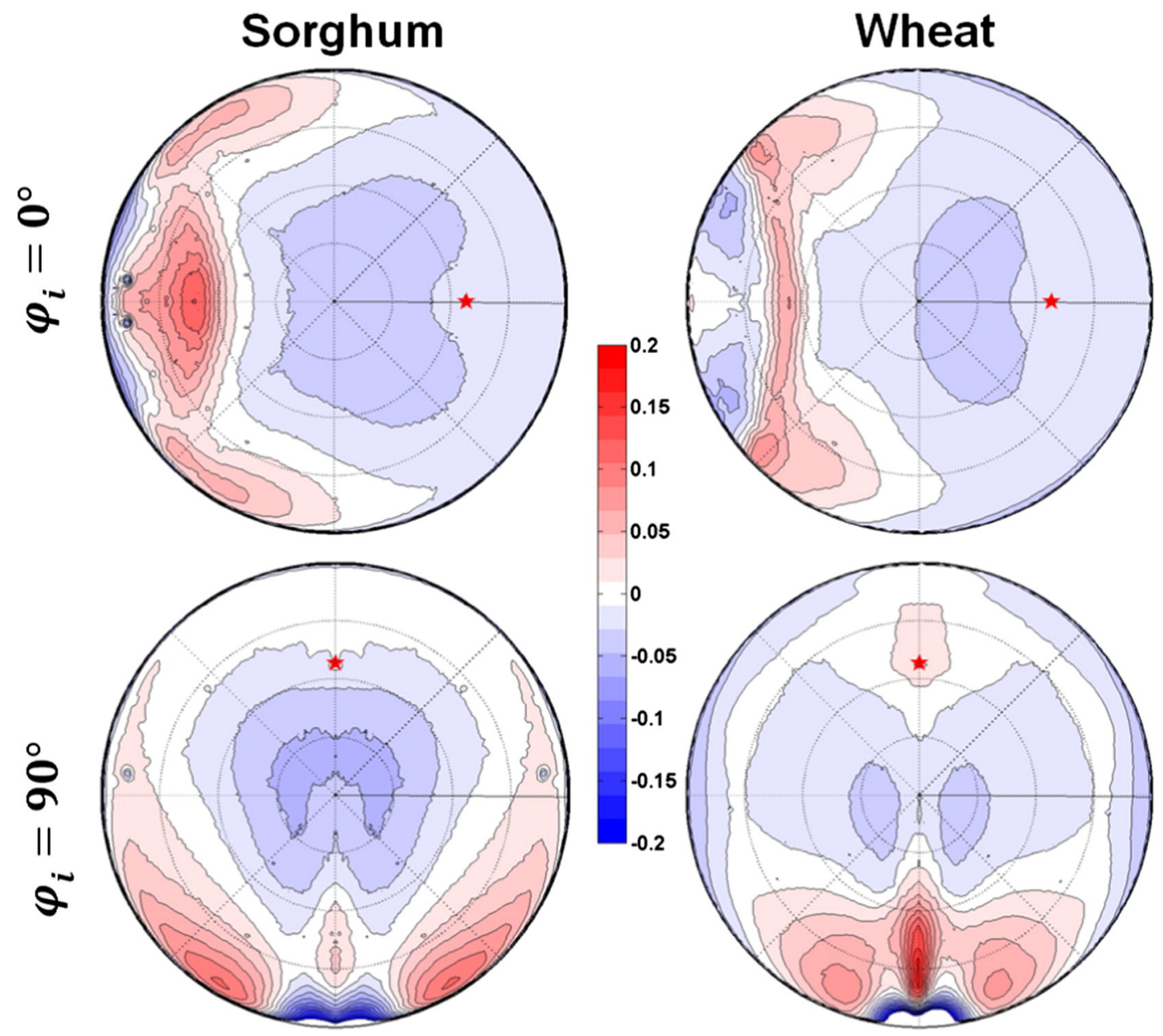

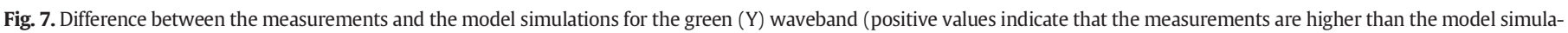
tions). (For interpretation of the references to color in this figure legend, the reader is referred to the web version of this article.)

Compared to the ACT model, the specular lobe seems to spread more in azimuth. The shape of the specular pattern is also different in the illumination plane; it has a more pronounced specular lobe. The facet specular assumption may be the most limiting hypothesis and thus mainly responsible for these discrepancies. As a matter of fact, it is highly probable that the diffuse component of BRDF is not completely Lambertian but is affected by the surface microstructure. The backward scattering as modeled through the ACT model is almost negligible.

\section{Conclusion}

The anisotropy of monocot leaves was already observed by Combes et al. (2007) and Comar et al. (2012). Our measurements on wheat and sorghum leaves confirm these results: the leaf appears more specular when illuminated perpendicular to the veins as compared to an illumination parallel to the veins. However, the DHRF of the leaf appears to be marginally dependent on the illumination azimuth: the smaller specular lobe in the principal plane for parallel illumination is compensated by an increase of the spread in azimuth, conversely to the thinner but more pronounced specular lobe for perpendicular illumination. A qualitative explanation is proposed, based on the macro-scale roughness of the leaf surface due to the presence of veins: the roughness depends on the azimuth of the veins, with a maximum in a direction perpendicular to the veins.

Beyond these qualitative explanations, the ACT model, a physically based BRDF model was proposed in this paper to obtain a description of the physical processes. It corresponds to an extension of the azimuthally isotropic model of Cook and Torrance (1981) already used by Bousquet et al. (2005) to describe dicotyledonous leaf BRDF (the CT model). The ACT model considers two orthogonal components for the roughness as suggested by Ward (1992). The ACT model includes four parameters to describe the surface reflectance, a) the refraction index, b) the roughness parallel to the veins, $c$ ) the perpendicular roughness and d) the volume scattering $D H R F_{\text {diff }}$ approximated as Lambertian and independent from the illumination azimuth. The parameters of the ACT model have been adjusted to achieve a good agreement between the simulated and measured reflectance values.

As expected, the estimated refraction index was found independent from the wavelength, with values around $n \approx 1.32$ both for wheat and sorghum, which is in the range of values found in previous studies. However, values estimated for the refraction index may partly be compensated by the Fresnel and shadowing-masking terms as already pointed out by Ward (1992). This makes it more difficult to arrive at a precise and accurate estimation of the parameters. The estimated values of the roughness parameters are in agreement with the range found in the literature for leaves (Bousquet et al., 2005) as well as some construction materials (Ward, 1992). Roughness in the direction perpendicular to the veins is about twice that parallel to the veins. However, roughness values were found to be lower for wheat as compared to sorghum, converse to what was expected from the scanning electronic microscope images showing a higher roughness at the macro-scale for wheat. This finding is also confirmed by the much stronger backscattering hotspot feature observed for wheat for perpendicular illumination - consistent 
with a rougher wheat leaf surface at the macro-scale level. Artifacts in the roughness estimates may come from the specific distribution of facets orientations used. More realistic facet distributions could be directly derived from the BRF measurements without any assumption on a parametric distribution law such as suggested by Obein, Leroux, Knoblauch and Viénot (2003) and Ngan, Durand and Matusik (2005). Furthermore, facet distributions may be alternatively derived from leaf surface topography measurements using atomic force microscopy (Giessibl, 2003). Possible multi-scale roughness features could also partly explain the uncertainties in the retrieved roughness values. The addition of a diffuse component to the micro-facet scattering properties suggested by Oren and Nayar (1994) and Simonot (2009) would improve the description of the diffuse component with the simulation of the backscattering directional features. The simple shadowingmasking function proposed by Blinn (1977) could be improved using the Ashikmin et al. (2000) formulation. In addition, the volume scattering was assumed Lambertian and independent from the illumination azimuth which is probably too crude an approximation. Further work is therefore needed to get more realistic leaf BRDF simulations using the several improvements suggested above.

Although not perfect, the proposed ACT model allows describing the main BRDF features of monocot leaves with a small number of parameters. It provides reasonable performance at the BRF level and better at the DHRF level. However, the validation of this model should be extended to other zenith incidence angles as well as to the near infrared domain where the volume scattering component will be much stronger than in the visible domain.

The proposed ACT model may be used to optimize the measurement configuration and the data pre-processing (Barnes, Dhanoa, \& Lister, 1989) when targeting leaf biochemical composition from the widely used non-destructive chemometric techniques (Preys, Roger, \& BoUlet, 2008). Clearly, the effects corresponding to the surface features need to be minimized to better capture the contribution from the volume scattering that vehicles the biochemical information. The ACT model may be also used to evaluate the importance of the non-Lambertian nature of leaves when simulating canopy reflectance in the case of azimuthally isotropic leaves, as proposed by Stuckens et al. (2009). However, very few canopy radiative transfer models are able to incorporate such a detailed description of the leaf BRDF, calling thus for further developments.

\section{Acknowledgment}

We would like to thank Bernard Noël, Jean Christophe Ribes and all the personnel of the Muséum d'Histoire Naturelle greenhouse who kindly helped us to use their facility in order to allow us to perform our study. Many thanks as well to Gwenaelle Barillet who grew the wheat plants; to Oulfa Belhadj-Khlaifi who helped us acquire MEB pictures; to Andrew Ratner and Shawn Carlisle Kefauver who corrected the English of this manuscript. This study was also partly supported by the Programme National de Teledetection Spatiale as well as by Arvalis-Institut du Végétal through the funding of Alexis Comar PhD grant.

\section{References}

Ashikmin, M., Premoze, S., \& Shirley, P. (2000). A microfacet-based BRDF generator. Proceedings of the 27th annual conference on Computer graphics and interactive techniques (pp. 65-74) (Available at: http://dl.acm.org/citation.cfm?id=344814, Accessed December 10, 2012)

Barnes, R. J., Dhanoa, M. S., \& Lister, S. J. (1989). Standard normal variate transformation and de-trending of near-infrared diffuse reflectance spectra. Applied Spectroscopy, 43(5), 772-777.

Beckmann, P., \& Spizzichino, A. (1963). The scattering of electromagnetic waves from rough surfaces. New York: Macmillan: London, England:Pergamon Press.

Blinn, J. F. (1977). Models of light reflection for computer synthesized pictures. ACM SIGGRAPH Computer Graphics (pp. 192-198) (Available at: http://dl.acm.org/ citation.cfm?id=563893, Accessed September 3, 2012)
Bousquet, L., Lachérade, S., Jacquemoud, S., \& Moya, I. (2005). Leaf BRDF measurements and model for specular and diffuse components differentiation. Remote Sensing of Environment, 98(2-3), 201-211.

Brakke, T., Smith, J., \& Harnden, J. (1989). Bidirectional scattering of light from tree leaves. Remote Sensing of Environment, 29(2), 175-183.

Breece Iii, H. T., \& Holmes, R. A. (1971). Bidirectional scattering characteristics of healthy green soybean and corn leaves in vivo. Applied Optics, 10(1), 119-127.

Chelle, M. (2006). Could plant leaves be treated as Lambertian surfaces in dense crop canopies to estimate light absorption? Ecological Modelling, 198(1-2), 219-228.

Comar, A., Baret, F., Viénot, F., de Yan, L., \& Solan, B. (2012). Wheat leaf bidirectional reflectance measurements: Description and quantification of the volume, specular and hot-spot scattering features. Remote Sensing of Environment, 121, 26-35.

Combes, D., Bousquet, L., Jacquemoud, S., Sinoquet, H., Varlet-Grancher, C., \& Moya, I. (2007). A new spectrogoniophotometer to measure leaf spectral and directional optical properties RID F-8842-2010. Remote Sensing of Environment, 109(1), 107-117.

Cook, R. L., \& Torrance, K. E. (1981). A reflectance model for computer graphics. ACM SIGGRAPH Computer Graphics (pp. 307-316).

Fliege, J., \& Maier, U. (1999). The distribution of points on the sphere and corresponding cubature formulae. IMA Journal of Numerical Analysis, 19(2), 317-334.

Fresnel, A. (1868). Oeuvres complètes d'Augustin Fresnel. Available at: http://books google.com/books?hl=en\&lr=lang_en|lang_fr\&id =KyaYIExTITwC\&oi=fnd\&pg $=$ PA1\&ots $=$ S3h0RijJCS\&sig $=$ BE8USqiX-QRvavykvBLin8i8fZs (Accessed February 4, 2013)

Giessibl, F. J. (2003). Advances in atomic force microscopy. Reviews of Modern Physics, 75(3), 949.

Howard, J. A. (1971). Luminance and Luminous Intensity Indicatrices of Isobilateral Leaves. Applied Optics, 10(10), 2354-2360.

Jacquemoud, S., \& Baret, F. (1990). Prospect a Model of Leaf Optical-Properties Spectra. Remote Sensing of Environment, 34(2), 75-91.

Kurt, M., Szirmay-Kalos, L., \& Kvrivánek, J. (2010). An anisotropic brdf model for fitting and monte carlo rendering. ACM SIGGRAPH Computer Graphics, 44(1), 3.

Levizou, E., Drilias, P., Psaras, G. K., \& Manetas, Y. (2005). Nondestructive assessment of leaf chemistry and physiology through spectral reflectance measurements may be misleading when changes in thricome density co-occur. New Phytologist, 165(2) 463-472.

Lewis, P. (2007). 3D canopy modelling as a tool in remote-sensing research. Frontis, 22, 219-229.

Marschner, S. R., Westin, S. H., Lafortune, E. P. F., \& Torrance, K. E. (2000). Image-based bidirectional reflectance distribution function measurement. Applied Optics, 39(16), 2592-2600.

Nayar, S. K., Ikeuchi, K., \& Kanade, T. (1991). Surface reflection: Physical and geometrical perspectives. IEEE Transactions on Pattern Analysis and Machine Intelligence, 13(7), 611-634.

Nelder, J. A., \& Mead, R. (1965). A simplex method for function minimization. The Computer Journal, 7(4), 308-313.

Ngan, A., Durand, F., \& Matusik, W. (2005). Experimental analysis of BRDF models. Proceedings of the Eurographics Symposium on Rendering (pp. 117-226) (Available at: http://www.merl.com/reports/docs/TR2005-151.pdf, Accessed December 10 2012)

Nicodemus, F. E., Richmond, J. C., Hsia, J. J., Ginsberg, I. W., \& Limperis, T. (1977) Geometrical considerations and nomenclature for reflectance. : US Dept. of Commerce, National Bureau of Standards: for sale by the Supt. of Docs., US Govt. Print. Off (Vol. 160).

Obein, G., Leroux, T., Knoblauch, K., \& Viénot, F. (2003). Visually relevant gloss parameters. 11th International Metrology Congress, Toulon, France (pp. 20-24).

Oren, M., \& Nayar, S. K. (1994). Generalization of Lambert's reflectance model. Proceedings of the 21st annual conference on computer graphics and interactive techniques (pp. 239-246) (Available at: http://dl.acm.org/citation.cfm?id=192213, Accessed April 3, 2013)

Papetti, T. J., Walker, W. E., Keffer, C. E., \& Johnson, B. E. (2007). Coherent backscatter: Measurement of the retroreflective BRDF peak exhibited by several surfaces relevant to ladar applications. Proc. SPIE6682 E (pp. 66820E-13) (66820).

Preys, S., Roger, J. M., \& BoUlet, J. C. (2008). Robust calibration using orthogonal projection and experimental design. Application to the correction of the light scattering effect on turbid NIR spectra. Chemometrics and Intelligent Laboratory Systems, 91(1), 28-33.

Rense, W. A. (1950). Polarization studies of light diffusely reflected from ground and etched glass surfaces. Journal of the Optical Society of America, 40(1), 55-56.

Sarto, A. W., Woldemar, C. M., \& Vanderbilt, V. C. (1990). In C. J. Hailey, \& O. H. W. Siegmund (Eds.), Polarized Light Angle Reflectance Instrument I Polarized Incidence (POLAR:I) (pp. 220-230) (Available at: http://spie.org/x648.html?product_id= 962893, Accessed October 18, 2012)

Simonot, L. (2009). Photometric model of diffuse surfaces described as a distribution of interfaced Lambertian facets. Applied Optics, 48(30), 5793-5801.

Smith, B. (1967). Geometrical shadowing of a random rough surface. IEEE Transactions on Antennas and Propagation, 15(5), 668-671.

Sparrow, E. M., \& Cess, R. D. (1978). Radiation heat transfer. Series in thermal and fluids engineering. New York: McGraw-Hill (Augmented ed., 1. Available at: http://adsabs. harvard.edu/abs/1978rht..book.....S, Accessed August 2, 2013)

Stuckens, J., Somers, B., Delalieux, S., Verstraten, W. W., \& Coppin, P. (2009). The impact of common assumptions on canopy radiative transfer simulations: A case study in Citrus orchards. Journal of Quantitative Spectroscopy E' Radiative Transfer, 110(1-2), $1-21$.

Torrance, K. E., \& Sparrow, E. M. (1967). Theory for off-specular reflection from roughened surfaces. Journal of the Optical Society of America, 57(9), 1105-1114. 
Vanderbilt, V. C., \& Grant, L. (1986). Polarization photometer to measure bidirectional reflectance factor $\mathrm{R}(55,0 ; 55,180)$ of leaves. Optical Engineering 25(4), 254566

Vigneau, N., Ecarnot, M., Rabatel, G., \& Roumet, P. (2011). Potential of field hyperspectra imaging as a non destructive method to assess leaf nitrogen content in Wheat. Field Crops Research, 122(1), 25-31.
Ward, G. J. (1992). Measuring and modeling anisotropic reflection. Computer Graphics, 26(2), 265-272.

Weidner, V. R., \& Hsia, J. J. (1981). Reflection properties of pressed polytetrafluoroethylene powder. JOSA, 71(7), 856-861.

Woolley, J. T. (1971). Reflectance and transmittance of light by leaves. Plant Physiology, 47(5), 656-662. 